\title{
INFLUÊNCIA DA ADIÇÃO DE LUBRIFICANTES E SÓLIDOS INERTES NAS PROPRIEDADES FISICAS DE FLUIDOS DE PERFURAÇÃO ARGILOSOS
}

\author{
Jeniffer Silva Martins ' \\ Renalle Cristina Alves de Medeiros Nascimento ${ }^{2}$ \\ Luciana Viana Amorim '
}

\section{Resumo}

Durante a perfuração de poços, a incorporação excessiva de sólidos aos fluidos de perfuração promove o aumento dos custos operacionais. Em vista disso, este trabalho tem como objetivo principal avaliar as propriedades dos fluidos de perfuração após a incorporação de sólidos inertes. Para tanto, foi realizado um estudo dividido em duas etapas, na primeira etapa, os fluidos apresentavam em sua composição apenas a argila bentonítica sódica, enquanto que na segunda etapa, eram compostos por argila bentonítica sódica e lubrificante. Os fluidos tiveram amostras de carbonato de cálcio e areia fina incorporados na sua composição. Foram avaliadas a densidade e propriedades, como reologia, filtração, lubricidade e o coeficiente de prisão diferencial. Os resultados evidenciaram que: i) a adição dos contaminantes aos fluidos provocou alterações em todas as propriedades estudadas ii) a carbonato de cálcio não atuou eficientemente como agente obturante e iii) a presença do lubrificante no fluido promoveu melhorias nas propriedades de lubricidade e prisão diferencial, de forma que a incorporação de sólidos ao fluido não afetou de maneira significativa tais propriedades.

Palavras-chave: Fluidos argilosos; Incorporação de sólidos; Lubricidade.

\section{INFLUENCE OF THE ADDITION OF THE LUBRICANT AND INERTS SOLIDS IN PHYSICAL PROPERTIES OF DRILLING MUDS}

\begin{abstract}
During the well drilling operation, the excessive incorporation of the solids to the drilling fluids promotes increased operating costs. Based on that, this study has as main objective to evaluate the properties of drilling fluids after addition of inert solids. It was performed a study divided in two stages, in the first stage, the fluids had in their composition only sodium bentonite clay, while in the second stage the fluids were composed of sodium bentonite clay and lubricants. The fluids incorporated in their composition calcium carbonate and fine sand samples. The density and the properties such as, rheology, filtration, lubricity and differential sticking coefficient have been evaluated. The results showed that: i) the addition of contaminants to the fluids caused changes in all studied properties ii) calcium carbonate did not act efficiently as bridging agente and iii) the presence of the lubricant in the fluid promoted improvements in the properties of lubricity and differential sticking, that incorporation of solids to the fluids does not significantly affect such properties.
\end{abstract}

Keywords: Drilling muds; Solids incorporation; Lubricity.

\section{INTRODUÇÃO}

Durante a perfuração de poços de petróleo, sólidos originados pela quebra da broca incorporam-se gradualmente ao fluido de perfuração. Até certo ponto, essa incorporação é aceitável e não prejudica o desempenho do fluido, porém quando se tem sólidos em excesso ocorre uma perda das propriedades requeridas. Esse excesso de sólidos é uma

'Departamento de Engenharia Mecânica, Universidade Federal de Campina Grande - UFCG, Campina Grande, PB, Brasil.

E-mail: Jeniffersmartins@gmail.com

${ }^{2}$ Programa de Recursos Humanos, Universidade Federal de Campina Grande - UFCG, Campina Grande, PB, Brasil. 
consequência de processos insatisfatórios de limpeza dos fluidos ou é ocasionado pela presença de grande quantidade de sólidos ativos [I].

Entende-se como sólidos ativos aqueles que conseguem se hidratar em água e favorecem uma formação de uma estrutura gel no fluido, como por exemplo a argila. Em contrapartida, os sólidos inertes são sólidos eletricamente neutros, que não incham e de fácil dispersão, consistem de materiais como areia, carbonato de cálcio, barita, entre outros [2]. Os sólidos inertes são responsáveis por grandes problemas na perfuração, por este motivo, são necessários estudos sobre a incorporação de sólidos aos fluidos de perfuração e os efeitos que estes ocasionam nas propriedades requeridas para um desempenho eficiente, de modo a minimizar as alterações decorridas deste processo e assim garantir melhor controle sobre as propriedades dos fluidos.

Um dos problemas causados pela presença de sólidos inertes no fluido é a elevação da pressão hidrostática do poço, ocasionada pelo aumento da densidade do fluido. Isso proporciona um aumento na quantidade de fluido que invade a formação [3]. Além do mais, quando se tem sólidos inertes incorporados ao fluido, o reboco formado durante o processo de invasão é mais espesso, isso porque essas partículas tendem a se depositar na parede do poço, reduzindo o seu diâmetro e aumentando as possibilidades de ocorrer algum problema, como por exemplo, a prisão diferencial [4].

A prisão diferencial é causada por um diferencial de pressão, que ocorre quando a coluna de fluido de perfuração exerce pressão demasiada na coluna de perfuração, pressionando-a sobre o reboco que está depositado em uma formação permeável [5]. Como resultado a ferramenta de perfuração fica imobilizada, mas a circulação do fluido é mantida normalmente. Esse fenômeno é indesejável, pois gera grandes prejuízos técnicos e econômicos, a exemplo da suspensão das atividades da sonda ou até mesmo o abandono do poço [6].

Muitos estudos mostram que a adição de determinados lubrificantes em fluidos argilosos reduz o risco de prisão diferencial, embora ainda possa haver a prisão de tubos, a força necessária para liberá-lo é bastante reduzida [7]. Isso porque os lubrificantes são substâncias que colocadas entre duas superfícies formam uma película protetora que tem como função principal função reduzir o atrito, o desgaste, auxiliar no controle da temperatura, proporcionando a limpeza do equipamento, protegendo contra a corrosão decorrente dos processos de oxidação, podendo também, ser agente de transmissão de força e movimento [8].

É bastante comum a perfuração de arenitos e calcários no setor petrolífero, assim diante do exposto, esse trabalho tem por objetivo principal avaliar os efeitos que a incorporação de sólidos, originados durante a perfuração desses tipos de rocha, ocasionam nas propriedades dos fluidos, e verificar se a adição de lubrificante, em fluidos que foram contaminados com esses sólidos, se mostra eficiente para reduzir o risco de prisão por diferencial.

\section{MATERIAIS E MÉTODOS}

\section{I Materiais}

Foram utilizados os seguintes materiais para o estudo:

- Uma amostra de argila bentonítica sódica, conhecida comercialmente como Brasgel PA. Esta argila é do tipo esmectita, quimicamente modificada com sal sódico e fornecida pela Empresa Bentonit União Nordeste Ltda - BUN, situada em Campina Grande/PB;

- Lubrificante de origem vegetal de alta lubricidade hidratado quimicamente com ácidos e neutralizantes alcalinos, na concentração de $1 \%$;

- Carbonato de cálcio com diâmetro médio de 13,88 $\mu \mathrm{m}$ fornecido pela PETROBRAS e utilizado como contaminante;

- Areia fina fornecida pela PETROBRAS e utilizada como contaminante.

O estudo foi dividido em duas etapas. Na primeira etapa, os fluidos estudados apresentaram em sua composição apenas argila e foram denominados de FI a F7. Na segunda etapa, os fluidos denominados de $\mathrm{F} 8$ a F 4 apresentam argila e lubrificante em sua composição.

A incorporação de sólidos aos fluidos estudados se deu de acordo com a Tabela I. Assim, os fluidos FI e F8; F2 e F9; F3 e FIO; F4 e FII; F5 e FI2; F6 e FI3; F7 e FI4 apresentam a mesma concentração de sólidos.

\subsection{Metodologia}

Na primeira etapa do estudo, os fluidos foram preparados com água e argila e tiveram amostras de areia e carbonato de cálcio incorporados na sua composição ( FI a F7). Enquanto que na segunda etapa, além dos mesmos procedimentos adotados na primeira, um lubrificante de origem vegetal foi adicionado aos fluidos (F8 a Fl4).

Os fluidos argilosos foram preparados em um agitador mecânico da marca Hamilton Beach, modelo 936, a argila foi adicionada à água deionizada e mantida sob agitação

Tabela I. Matriz de planejamento utilizada para as duas etapas realizadas no estudo

\begin{tabular}{lcc}
\hline Fluidos & Concentração de Areia & $\begin{array}{c}\text { Concentração de } \\
\text { Carbonato de Cálcio }\end{array}$ \\
\hline FI/F8 & $-\mathrm{I}(0 \mathrm{~g} / 350 \mathrm{~mL})$ & $-\mathrm{I}(0 \mathrm{~g} / 350 \mathrm{~mL})$ \\
F2/F9 & + I $(10 \mathrm{~g} / 350 \mathrm{~mL})$ & $-\mathrm{I}(0 \mathrm{~g} / 350 \mathrm{~mL})$ \\
F3/FI0 & $-\mathrm{I}(0 \mathrm{~g} / 350 \mathrm{~mL})$ & $+I(10 \mathrm{~g} / 350 \mathrm{~mL})$ \\
F4/FI I & + I $(10 \mathrm{~g} / 350 \mathrm{~mL})$ & + I $(10 \mathrm{~g} / 350 \mathrm{~mL})$ \\
F5/FI2 & $0(5 \mathrm{~g} / 350 \mathrm{~mL})$ & $0(5 \mathrm{~g} / 350 \mathrm{~mL})$ \\
F6/FI3 & $0(5 \mathrm{~g} / 350 \mathrm{~mL})$ & $0(5 \mathrm{~g} / 350 \mathrm{~mL})$ \\
F7/FI4 & $0(5 \mathrm{~g} / 350 \mathrm{~mL})$ & $0(5 \mathrm{~g} / 350 \mathrm{~mL})$ \\
\hline
\end{tabular}


constante (25.000 rpm), durante 20 minutos. Em seguida, o fluido foi transferido para um recipiente fechado e colocado em repouso por 24 horas. Esse tempo em repouso é conhecido como tempo de cura e é essencial para permitir a total hidratação das partículas de argila.

Após 24 horas, os sólidos (areia e carbonato de cálcio) foram pesados em balança analítica de alta precisão e em seguida, utilizando $o$ agitador mecânico, foram adicionados ao fluido, sob agitação constante (17.000 rpm). Após a incorporação de todo o sólido, os fluidos permaneceram durante 5 minutos sob agitação na velocidade de $25.000 \mathrm{rpm}$. Aqueles fluidos cuja composição continham lubrificante, tiveram este adicionado, e foram em seguida submetidos a uma agitação por 5 minutos.

Logo em seguida foi determinada a densidade dos fluidos, através da balança de lama modelo 204 Fann de acordo com a norma API [9]. Para determinar as propriedades reológicas dos fluidos de perfuração foi utilizado um viscosímetro rotativo Fann 35A. O estudo reológico foi realizado de acordo com a norma API [9]. Foram realizadas leituras nas velocidades de 600, 300, 200, 100, 6 e $3 \mathrm{rpm}$. Para obtenção da força gel inicial $\left(G_{o}\right)$, o viscosímetro foi acionado a velocidade de $600 \mathrm{rpm}$ por 15 segundos, em seguida permaneceu em repouso por 10 segundos e efetuada a leitura em $3 \mathrm{rpm}$. Para a leitura da força gel final $\left(G_{f}\right)$, foi feito o mesmo procedimento do $G_{o}$, mas com repouso de 10 minutos. A viscosidade aparente (VA) e plástica (VP), o limite de escoamento (LE) e a força gel (FG) foram calculados utilizando as Equações I, 2, 3 e 4, respectivamente.

- Viscosidade aparente (VA):

$V A=\frac{\mathrm{L}_{600}}{2}(\mathrm{cP})$

- Viscosidade plástica (VP):

$V P=L_{600}-L_{300}(\mathrm{cP})$

- Limite de escoamento (LE):

$L E=L_{300}-V P\left(\mathrm{~N} / \mathrm{m}^{2}\right)$

- Força Gel (FG):

$$
F G=G_{f}-G_{0}\left(\mathrm{~N} / \mathrm{m}^{2}\right)
$$

Para realização do estudo de lubricidade foi necessário agitar os fluidos por 5 minutos, em seguida, foi utilizado um lubrifimetro OFITE, que teve o torque inicial e a velocidade ajustados para zero e $60 \mathrm{rpm}$, respectivamente. Em seguida foi lentamente aplicada uma força de $150 \mathrm{lbf}$, durante 5 minutos e só então, a leitura do torque exercido pelo fluido foi efetuada. O cálculo do fator de correção (FC) é realizado por meio da leitura referente ao torque da água, de acordo com a Equação 5 e o coeficiente de lubricidade $(\mathrm{CL})$ de acordo com a Equação 6.

$$
\begin{aligned}
& F C=\frac{34,0}{\text { Leitura }_{\text {agua }}} \\
& C L=\frac{\text { FC x Leitura }}{\text { obtida }} \\
& 100
\end{aligned}
$$

Após determinar o coeficiente de lubricidade, foi realizado um ensaio com o objetivo de determinar o coeficiente de prisão diferencial (CPD) e o volume do filtrado $\left(\mathrm{VF}_{\mathrm{PD}}\right)$ no equipamento Differential Sticking Tester da marca Fann. Os fluidos inicialmente foram agitados durante 5 minutos, posteriormente, foram introduzidos no interior da célula do equipamento Differential Sticking Tester da marca Fann. Em seguida, foram submetidos a uma pressão de aproximadamente 477,5 psi durante 10 minutos, para que o reboco fosse formado. Ao final dos 10 minutos foi medido o volume de filtrado $\left(\mathrm{VF}_{\mathrm{PD}}\right)$. Após esse período, utilizou uma alavanca para baixar o flat plate, que foi mantido nessa posição por 2 minutos. Então, aguardou mais 10 minutos para efetuar as seis leituras de toque com o auxílio de um torquímetro acoplado ao flat plate.

Com os valores obtidos do torque, foi calculado o torque médio $\left(\mathrm{T}_{\mathrm{m}}\right)$, que consiste de uma média aritmética $\mathrm{e}$ em seguida o coeficiente de tendência de prisão diferencial (CPD) foi determinado de acordo com a Equação 7:

$$
C P D=\frac{\mathrm{T}_{\mathrm{m}}}{1000}
$$

\section{RESULTADOS E DISCUSSÃO}

\section{I Densidade}

A densidade $(\rho)$ do fluido de perfuração é uma propriedade importante e deve ser monitorada de modo que a pressão hidrostática $\left(P_{h}\right)$ seja suficientemente alta para controlar os fluidos da formação, porém sem ultrapassar os limites que possam causar danos, como o faturamento da formação rochosa ou perdas de circulação. É importante que o teor de sólidos nos fluidos seja mantido o mínimo possível, uma vez que, irá influenciar de forma direta na sua densidade. As densidades dos fluidos estudados, na primeira e na segunda etapa, estão apresentadas na Figura I.

É possível observar através das Figuras I e 2, que a incorporação dos sólidos resultou em aumento da densidade. Os fluidos sem sólidos incorporados (FI e F8) apresentaram valores de densidade equivalentes a $\mathrm{I}, 025 \mathrm{~g} / \mathrm{cm}^{3} \mathrm{e} \mathrm{I}, 02 \mathrm{~g} / \mathrm{cm}^{3}$, respectivamente, enquanto que os fluidos com sólidos incorporados apresentaram valores acima de $1,03 \mathrm{~g} / \mathrm{cm}^{3}$.

O aumento da quantidade de sólidos incorporados promoveu um acréscimo progressivo desta propriedade. Os fluidos com maiores concentrações de sólidos ( $\mathrm{F} 4 \mathrm{e} \mathrm{FII}$ ), cerca de 20 gramas ( $10 \mathrm{~g}$ de areia e $10 \mathrm{~g}$ de carbonato de cálcio), apresentaram densidades maiores que os demais, correspondente a I, $06 \mathrm{~g} / \mathrm{cm}^{3}$ e I, $05 \mathrm{~g} / \mathrm{cm}^{3}$, respectivamente. 


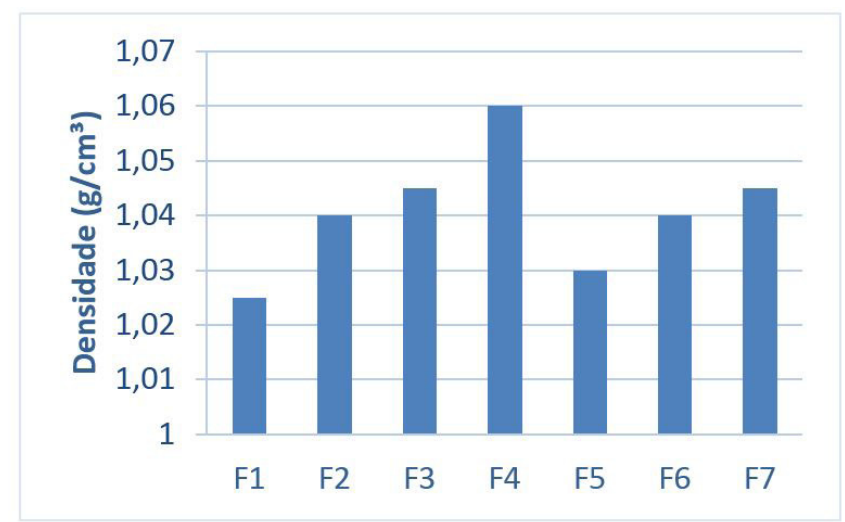

(a)

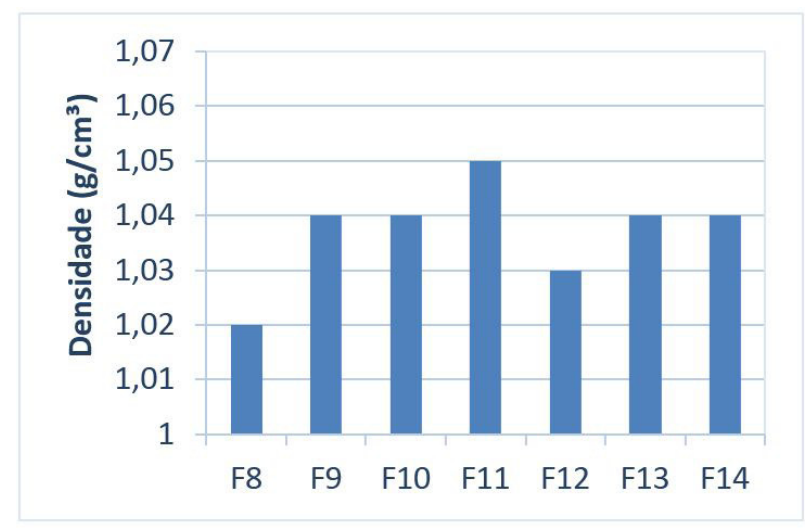

(b)

Figura I. Densidades dos fluidos estudados. (a) Fluidos estudados na primeira etapa (sem lubrificante) (b) fluidos estudados na segunda etapa (com lubrificante).

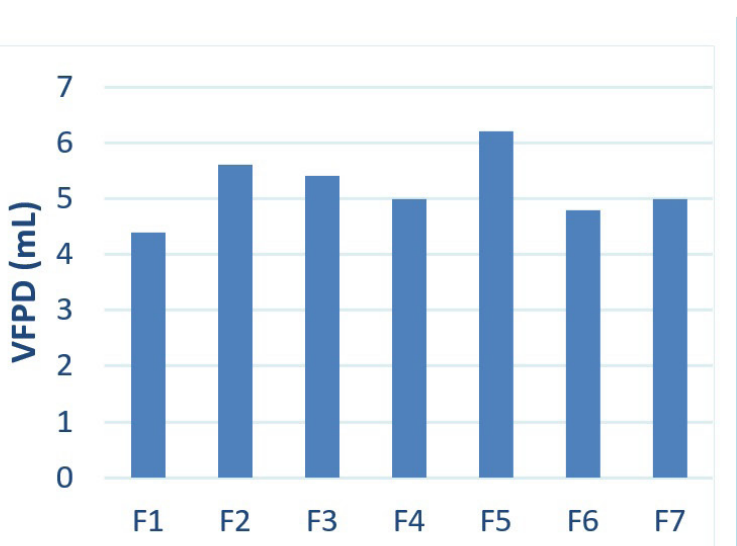

(a)

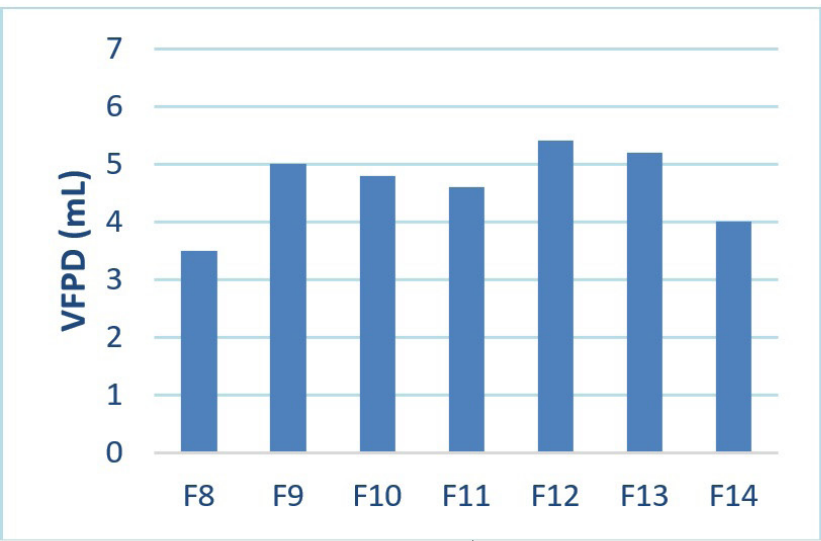

(b)

Figura 2. Volume do filtrado dos fluidos argilosos estudados. (a) Fluidos estudados na primeira etapa (sem lubrificante) (b) fluidos estudados na segunda etapa (com lubrificante).

Ao comparar os resultados obtidos, na primeira e na segunda etapa, é possível observar que a adição de lubrificantes ocasionou uma pequena redução nas densidades dos fluidos de perfuração.

De acordo com Pereira [10], para iniciar o alargamento do furo-guia (primeiros passos da perfuração de poços) é necessário que a densidade padrão dos fluidos argilosos estejam em torno de 4,8 a $7,2 \mathrm{~g} / \mathrm{cm}^{3}$, podendo esse valor ser dimensionado de acordo com a pressão de poros da formação. Os resultados obtidos foram bem inferiores, e se faz necessário o uso de aditivos que conferem densidade, como por exemplo a barita ou ilmenita. para adequar os fluidos estudados as especificações.

\subsection{Propriedades Reológicas}

Os resultados das propriedades reológicas dos fluidos estudados na primeira etapa estão apresentados na Tabela 2, enquanto que os da segunda etapa, na Tabela 3.
A incorporação dos sólidos inertes, tais como areia fina e carbonato de cálcio, provocaram uma alteração das propriedades reológicas dos fluidos de perfuração estudados. Foi observado que, maiores porcentagens de carbonato de cálcio e de areia resultaram no aumento dos valores da VA e LE, sendo o carbonato de cálcio, a variável de maior influência. Isso porque ao incorporar carbonatos de cálcio ao fluido de perfuração argiloso, os cátions multivalentes presentes irão reduzir as forças repelentes entre as partículas de argila e a película de água aderida, ocasionando a floculação das partículas de argila, o resultado disso é a gelificação de porções do fluido, com elevação abrupta da viscosidade e o aumento da força necessária para o escoamento do fluido [II].

Ao contaminar os fluidos com amostras de areia ou de carbonato de cálcio, foi observado que para aqueles sem lubrificantes (F2 e F3), a VP sofreu uma pequena variação, sendo a areia, o sólido de maior influência e para fluidos com lubrificante (F9 e FI0), a VP se manteve constante. 
Tabela 2. Propriedades reológicas dos fluidos estudados na primeira etapa (sem lubrificantes)

\begin{tabular}{|c|c|c|c|c|}
\hline Fluidos & VA (cP) & VP (cP) & LE $\left(\mathrm{N} / \mathrm{m}^{2}\right)$ & $F G\left(N / m^{2}\right)$ \\
\hline FI & 17,5 & 3,0 & 29,0 & 25,0 \\
\hline F2 & 16,5 & 4,0 & 25,0 & 22,0 \\
\hline F3 & 21,0 & 1,0 & 40,0 & 31,0 \\
\hline F4 & 22,5 & 7,0 & 31,0 & 25,0 \\
\hline F5 & 17,0 & 4,0 & 26,0 & 25,0 \\
\hline F6 & 18,5 & 3,0 & 31,0 & 24,0 \\
\hline F7 & 17,5 & 3,0 & 29,0 & 28,0 \\
\hline
\end{tabular}

VA- Viscosidade Aparente; VP- Viscosidade Plástica; LE- Limite de Escoamento; FG- Força Gel.

Tabela 3. Propriedades reológicas dos fluidos estudados na segunda etapa (com lubrificantes)

\begin{tabular}{|c|c|c|c|c|}
\hline Fluidos & VA (cP) & VP (cP) & LE $\left(\mathbf{N} / \mathbf{m}^{2}\right)$ & $F G\left(N / m^{2}\right)$ \\
\hline F8 & 36,0 & 4,0 & 64,0 & $\mathrm{I}, 0$ \\
\hline F9 & 38,5 & 4,0 & 69,0 & 5,0 \\
\hline FIO & 40,0 & 4,0 & 72,0 & 2,0 \\
\hline FII & $4 I, 5$ & 11,0 & 61,0 & 5,0 \\
\hline FI2 & 42,5 & 4,0 & 77,0 & 3,0 \\
\hline FI3 & 39,5 & 9,0 & 61,0 & 3,0 \\
\hline FI4 & 37,0 & 3,0 & 68,0 & 2,0 \\
\hline
\end{tabular}

VA- Viscosidade Aparente; VP- Viscosidade Plástica; LE- Limite de Escoamento; FG- Força Gel.

Entretanto, quando houve a incorporação de uma grande porcentagem de amostras de areia e carbonato de cálcio combinados ( $\mathrm{F} 4 \mathrm{e} \mathrm{FI} \mathrm{I}$ ) foi constatado um aumento significativo no valor de VP.

Por meio de uma análise estatística foi observado que para a FG, fluidos contaminados com amostras contendo areia e carbonato de cálcio apresentam resultados estatisticamente mais significativos do que aqueles que possuíam apenas com um dos sólidos. Porém, para os fluidos sem lubrificantes, o carbonato de cálcio foi a variável de maior influência, enquanto que para os fluidos com lubrificante, a areia foi responsável por maiores mudanças nas propriedades dos fluidos.

É possível observar que a adição de lubrificantes ocasionou um aumento das propriedades reológicas estudadas, com exceção da FG. De acordo com estudos desenvolvidos por Nascimento et al. [12], é desejável que lubrificantes de origem vegetal, sejam solúveis em água, porém, eles podem formar pequenas gotículas, que quando adicionado aos fluidos contendo argila bentonítica age como dispersante, neutralizando as cargas presentes nas superfícies das partículas de argila. De modo geral, sua adição ocasionaria uma redução nos valores das propriedades reológicas. Porém, no estudo realizado observou-se o comportamento inverso e este se deve, muito provavelmente, ao fato do teor de lubrificante adicionado ser suficiente para atingir a sua concentração micelar crítica (c.m.c.).

Alguns umectantes agem como dispersantes e segundo Farias et al. [13], as moléculas de umectantes podem atrair ou repelir as partículas de argila e penetrar entre as camadas, podendo assim aumentar ou diminuir a estabilidade do sistema. Adição de umectantes à água tende a saturar todas as interfaces, de modo que a partir de uma concentração denominada concentração micelar crítica (c.m.c.) há a saturação do meio e a formação de micelas, ocorrendo variações bruscas nas propriedades físico-químicas da solução.

\subsection{Volume de Filtrado $\left(\mathrm{VF}_{\mathrm{PD}}\right)$}

Os fluidos de perfuração devem ser tratados para manter a permeabilidade do reboco a mais baixa possível a fim de manter um poço estável e minimizar a invasão do filtrado [14]. Assim sendo, deve haver um controle no que diz respeito a essa invasão. Na Figura 2 são apresentados os valores do volume de filtrado $\left(\mathrm{VF}_{\mathrm{PD}}\right)$ obtidos por meio do ensaio de Prisão Diferencial na primeira e segunda etapa do estudo.

Por meio dos dados obtidos, é possível observar que a adição de lubrificantes ocasionou uma pequena redução do $\mathrm{VF}_{\mathrm{PD}}$. Enquanto que, a incorporação de sólidos inertes, em ambas as etapas, provocou um aumento do $\mathrm{VF}_{\mathrm{PD}}$, sendo a areia a variável de maior influência.

O carbonato de cálcio geralmente é utilizado como agente obturante, responsável por obturar os poros da formação, reduzindo $\circ \mathrm{VF}_{\mathrm{PD}}$. No estudo realizado, a redução do $\mathrm{VF}_{\mathrm{PD}}$ provocada pela adição do carbonato de cálcio foi mínima, muito provavelmente, por causa da conformação das partículas de argila no fluido, que impediu que os sólidos de carbonato de cálcio conseguissem penetrar nos poros e assim efetuar uma ação obturante eficiente. 
Dependendo da composição do fluido de perfuração utilizado, maiores valores de $\mathrm{VF}_{\mathrm{PD}}$ podem conduzir a formação de rebocos mais espessos, que reduzem o diâmetro do poço e aumentam a área de contato entre o tubo e a parede do poço, o que intensifica os riscos de ocorrer prisão. É possível observar que os fluidos (F2 e F9) que tiveram apenas areia incorporado apresentaram maiores valores de volume de filtrado. Neste caso, o reboco depositado na parede do poço tem uma tendência de possuir uma maior espessura que do que o formado pelo carbonato de cálcio. Assim, a probabilidade de acontecer prisão por diferencial de pressão é maior quando se tem fluidos contaminados com areia do que com carbonato de cálcio.

\subsection{Prisão Diferencial e Lubricidade}

Os valores de coeficiente de lubricidade $(C L)$ e de prisão diferencial (CPD), para os fluidos estudados, sem e com lubrificante, estão apresentados nas Tabelas 4 e 5, respectivamente.

A partir dos dados obtidos, é possível observar que o $C L$ e o CPD não sofrem variações significativas com incorporação de sólidos inertes, sendo a areia a variável de maior influência, enquanto que a variação da concentração de carbonato de cálcio não alterou significativamente os resultados. Os fluidos lubrificantes apresentam valores do $\mathrm{CL}$ em torno de 0,15 [14]. Contudo, os fluidos estudados na primeira etapa não tinham características lubrificantes, por consequência apresentaram valores elevados do $\mathrm{CL}$. $A$ adição do lubrificante provocou uma redução do $\mathrm{CL}$,

Tabela 4. CPD e CL dos fluidos argilosos sem lubrificante

\begin{tabular}{ccc}
\hline Fluidos & CPD & CL \\
\hline FI & 0,118 & 0,41 \\
F2 & 0,198 & 0,29 \\
F3 & 0,142 & 0,37 \\
F4 & 0.174 & 0,36 \\
F5 & 0,155 & 0,41 \\
F6 & 0,000 & 0,33 \\
F7 & 0,159 & 0,37 \\
\hline
\end{tabular}

CPD-Coeficiente de Prisão Diferencial; CL- Coeficiente de Lubricidade

Tabela 5. CPD e CL dos fluidos argilosos com lubrificante

\begin{tabular}{ccc}
\hline Fluidos & CPD & CL \\
\hline F8 & 0,077 & 0,058 \\
F9 & 0,109 & 0,133 \\
FI0 & 0,074 & 0,099 \\
FII & 0.102 & 0,168 \\
FI2 & 0,064 & 0,107 \\
FI3 & 0,085 & 0,086 \\
FI4 & 0,083 & 0,104 \\
\hline
\end{tabular}

CPD- Coeficiente de Prisão Diferencial; CL- Coeficiente de Lubricidade conferindo lubricidade aos fluidos, assim fica evidenciado a eficiência do lubrificante utilizado.

Houve uma correlação entre o CL e o CPD, de forma que os fluidos com maiores características lubrificantes apresentaram uma menor tendência de ocasionar a prisão por diferencial. Logo, foi possível observar que a adição do lubrificante ocasionou também a diminuição dos valores do CPD. Os maiores valores de CPD foram obtidos para os fluidos ( $F 2$ e $F 9$ ) de maior $\mathrm{VF}_{\mathrm{PD}}$, isso pode ser consequência da maior espessura de reboco, uma vez que, a areia é considerada um sólido inerte, tendendo a se depositar na parede do poço, formado um reboco mais espesso, o que aumenta as possibilidades de ocorrer à prisão diferencial.

De maneira geral, no estudo realizado foi observado que a incorporação de areia e carbonato de cálcio aos fluidos de perfuração promoveram alterações, na densidade, no desempenho reológico, nas propriedades de filtração, no CPD e no $\mathrm{CL}$, embora que em algumas situações essas variações foram mínimas, sendo o carbonato de cálcio a variável que exerceu maior influência na variação da densidade e das propriedades reológicas e a areia nos valores do $\mathrm{VF}_{\mathrm{PD}}, \mathrm{CPD}$ e CL. Já a adição de lubrificantes promoveu um aumento das propriedades reológicas, com exceção da FG, além de reduzir $\circ \mathrm{CL}$ e $\circ \mathrm{CPD}$. Para um lubrificante possuir um bom desempenho é importante que ele não altere as demais propriedades do fluido, reduzindo somente as propriedades de lubricidade e o risco de ocorrer a prisão por diferencial de pressão.

\section{CONCLUSÕES}

Pode-se concluir que: i) A densidade aumentou com a incorporação de sólidos inertes, enquanto a adição de lubrificantes promoveu uma pequena redução desta propriedade; ii) A incorporação de sólidos inertes aos fluidos promoveu um aumento nas propriedades reológicas; iii) $A$ adição de lubrificantes promoveu o aumento das propriedades reológicas, com exceção da FG; iv) O volume de filtrado aumenta com a incorporação de sólidos, enquanto a adição de lubrificante promove uma pequena redução; v) $A$ areia foi a variável de maior influência no $C L$ e CPD, ocasionando maiores valores destas propriedades; vi) Os valores de CPD presentaram relação com os valores de $\mathrm{CL}$, assim quanto menor o $\mathrm{CL}$, menor o risco de haver prisão por diferencial de pressão.

\section{Agradecimentos}

Ao CNPq pelo apoio financeiro, à Empresa Bentonit União Nordeste Ltda - BUN pelo fornecimento da argila bentonítica, a PETROBRAS por fornecer os aditivos utilizados e ao PEFLAB/LABDES, em nome da professora Luciana Amorim pelo uso de suas instalações físicas. 


\section{REFERÊNCIAS}

I Carvalho AL. Estudo dos fluidos de perfuração e seus impactos relacionados às atividades da indústria de petróleo [Monografia de graduação]. Itajubá: Universidade Federal de Itajubá; 2005.

2 Annis RM, Smith VM. Drilling fluids technology. Irving: Exxon Company; 1996.

3 Silva, C.T. Desenvolvimento de fluidos de perfuração a base de óleos vegetais [monografia de graduação]. Natal: Universidade Federal do Rio Grande do Norte, 2003.

4 Farias KV. Influência de umectante aniônico na reologia e espessura do reboco de fluidos hidroargilosos [dissertação de mestrado]. Campina Grande: Universidade Federal de Campina Grande; 2005.

5 Simon K, Gaurina-Medimurec N, Pasic B. Drilling fluids differential sticking tendency. Zagreto: Rudarsko-geoloskonaftnizboornik; 2005.

6 Souza DAF, Elias RN, Alves JLD, Landau L, Coutinho ALGA, Lomba RFT et al. Modelagem computacional da prisão diferencial da coluna de perfuração em poços não convencionais. Vitória da Conquista: SOFTEC, 2003 [Acesso em 15 jan. 2015]. Disponível em: http://www.nacad.ufrj.br/ rnelias/papers/so ftec2003.pdf

7 Nascimento RCAM, Amorim LV, Lira DS, Lira HL. O fenômeno de prisão diferencial: uma revisão da literatura. Revista Eletrônica de Materiais e Processos, 2010;5(2), 76-87 [acesso em 4 fev. 2015]. Disponível em: www.dema. ufcg.edu.br/revista.

8 Gomes, H.O.; Oliveira J Fo. Metodologia de avaliação da lubricidade de óleo Diesel. In: Rede Metrológica do Estado de São Paulo. Encontro para a Qualidade de Laboratórios; 2005 Jun 7-9; São Paulo, Brasil. São Paulo: REMESP; 2005.

9 American Petroleum Institute - API. Petroleum and natural gas industries - field testing of drilling fluids - Part I: water-based fluids, ISO/WD I04I4-I e ISO TC 67/SC 3 N. Washington; 2005.

10 Pereira E. Aspectos práticos importantes dos fluidos de perfuração modernos. In: Associação Brasileira de Águas Subterrâneas. X Congresso Brasileiro de Águas Subterrâneas; 1998 Set 9-II; São Paulo, Brasil. São Paulo: Associação Brasileira de Águas Subterrâneas; 1998.

II Pereira E. O Uso de inibidores de argilas como solução de problemas em sondagens. In: Associação Brasileira de Águas Subterrâneas. XII Encontro Nacional de Perfuradores de Poços; 200I; Petrópolis, Brasil. São Paulo: Associação Brasileira de Águas Subterrâneas; 200I. Suplemento [acesso em 29 jan. 20I5]. Disponível em: http:// aguassubterraneas.abas.org/.

12 Nascimento RCAM, Amorim LV, Santana LNL. Avaliação de aditivos lubrificantes em fluidos aquosos para perfuração de poços de petróleo. Revista Eletrônica de Materiais e Processos, 2008;3(3):56-64 [acesso em 12 jun. 2015]. Disponível em: www.dema.ufcg.edu.br/revista.

13 Farias KV, Amorim LV, Ferreira HC, Pereira E. Estudo da reologia e espessura do reboco de fluidos de perfuração: influência de dispersantes e umectante aniônicos. Revista Cerâmica. 2006;52:306-312.

14 Caenn R, Darley HCH, Gray GR. Composition and properties of drilling and completion fluids. 6th ed. Houston: Gulf Publishing Company; 20II. 720 p.

Recebido em: 01 Dez. 2015

Aceito em: 18 Jul. 2016 